\title{
The Association Between Vitamin D and Type 2 Diabetes Mellitus Complicated with Non-Alcoholic Fatty Liver Disease: An Observational Cross-Sectional Study
}

\author{
Yuling Xing ${ }^{1,2}$, Tiantian Cheng ${ }^{1,3}$, Fei Zhou' ${ }^{1,2}$, Huijuan Ma $\mathbb{D}^{1,4,5}$ \\ 'Department of Endocrinology, Hebei General Hospital, Shijiazhuang, 050017, People's Republic of China; ${ }^{2}$ Graduate School of Hebei Medical \\ University, Shijiazhuang, 0500 17, People's Republic of China; ${ }^{3}$ Department of Internal Medicine, School of Clinical Medicine, North China University of \\ Science and Technology, Tangshan, 063210, Hebei, People's Republic of China; ${ }^{4}$ Hebei Key Laboratory of Metabolic Diseases, Hebei General Hospital \\ Shijiazhuang, Hebei, 05005I, People's Republic of China; ${ }^{5}$ Department of Internal Medicine, Hebei Medical University, Shijiazhuang, Hebei, 0500I7, \\ People's Republic of China
}

Correspondence: Huijuan Ma, Email huijuanma19@I63.com

Objective: To investigate the association between vitamin D deficiency and NAFLD risk in patients with type 2 diabetes mellitus (T2DM).

Methods: Overall, 434 patients with T2DM admitted to Hebei General Hospital from January 2019 to December 2019 were selected as the study subjects. According to abdominal ultrasound findings, patients were divided into the NAFLD group and the non-NAFLD group. Participants were divided into two study groups according to the 25-hydroxyvitamin D [25(OH)D] level. 25(OH)D deficiency was defined if $25(\mathrm{OH}) \mathrm{D}$ vitamin levels were $<20 \mathrm{ng} / \mathrm{mL}$. Chi-square test and one-way analysis of variance were used to compare groups. The relationship between $25(\mathrm{OH}) \mathrm{D}$ and NAFLD risk was analyzed using correlation and regression analyses. Furthermore, subgroup analyses were performed to verify the robustness of the results.

Results: The 25(OH)D level in patients with T2DM complicated by NAFLD was significantly lower than in patients with T2DM only. Vitamin D deficiency was highly prevalent among T2DM patients with NAFLD. This study suggested that vitamin D deficiency was an independent factor for developing NAFLD in patients with T2DM. T2DM patients with vitamin D deficiency had 2.045 times higher risk of developing NAFLD than those without vitamin D deficiency. Vitamin D deficiency was associated with high NAFLD preference in T2DM patients with BMI $>23 \mathrm{~kg} / \mathrm{m}^{2}$, but not those with BMI $\leq 23 \mathrm{~kg} / \mathrm{m}^{2}$. The significant correlation between vitamin D deficiency and NAFLD was found in participants with BMI $>23 \mathrm{~kg} / \mathrm{m}^{2}$, age $\leq 65$ years, without hypertension, $\mathrm{TG}<1.7 \mathrm{mmol} / 1$, HDL $\geq 1 \mathrm{mmol} / 1 \mathrm{in}$ men, $\geq 1.3 \mathrm{mmol} / 1$ in women, $\mathrm{HBA} 1 \mathrm{C} \leq 7 \%$, or females.

Conclusion: This study suggests that T2DM people with BMI $>23 \mathrm{~kg} / \mathrm{m}^{2}$ were more susceptible to NAFLD by vitamin D deficiency and that it is necessary to maintain optimal serum vitamin $\mathrm{D}$ levels in this population.

Keywords: type 2 diabetes mellitus, non-alcoholic fatty liver disease, vitamin D

\section{Introduction}

Non-alcoholic fatty liver disease (NAFLD) was characterized by a spectrum of pathologies ranging from simple hepatocyte steatosis to non-alcoholic steatohepatitis (NASH). As the disease progresses, it can lead to liver cirrhosis and even the liver cancer. ${ }^{1}$ In a recent meta-analysis of 86 studies, the global prevalence of NAFLD was estimated to be $25.24 \%$ in the general population, while several meta-analyses indicated that in type 2 diabetes mellitus (T2DM) patients the prevalence was double (54-59.67\%). ${ }^{2-4}$ The prevalence of NAFLD has reached as high as $90 \%$, particularly in T2DM patients with obesity. ${ }^{5}$ However, accumulating evidence indicated that the effects of NAFLD extend beyond the liver and are negatively associated with a range of chronic diseases, most notably cardiovascular disease (CVD), T2DM 
and chronic kidney disease (CKD). ${ }^{6}$ However, there was no definitive treatment for NAFLD other than lifestyle intervention. ${ }^{7}$ Vitamin D was a lipid-soluble vitamin involved primarily in bone metabolism, calcium and phosphate homeostasis and skeletal development. ${ }^{8} 25$-Hydroxyvitamin D $[25(\mathrm{OH}) \mathrm{D}]$ was the active form of vitamin $\mathrm{D} .{ }^{9}$ The evaluation of vitamin D status usually relied on detection of the serum $25(\mathrm{OH}) \mathrm{D}$ concentration. ${ }^{10}$ Several epidemiologic studies show that low serum levels of $25(\mathrm{OH}) \mathrm{D}$ were associated with adverse health outcomes, such as metabolic syndrome, diabetes, chronic liver disease, cancer, cardiovascular disease and all-cause mortality. ${ }^{11-16}$ Worldwide, vitamin $\mathrm{D}$ deficiency was one of the most important public health issues and its prevalence in general populations is approximately $36 \%$ in the United States, $61 \%$ in Canada, $92 \%$ in Northern Europe, $45-98 \%$ in Asia, 31\% in Australia and 56\% in New Zealand. ${ }^{17}$ A meta-analysis concluded that vitamin D deficiency was common in Asia based on data from 472 studies with 746,564 participants. ${ }^{18}$ Epidemiological evidence indicated that low 25(OH)D level was an independent risk factor for NAFLD. ${ }^{14,19}$ A recent meta-analysis indicated a significant association between low 25(OH)D level and NAFLD. ${ }^{20}$ T2DM patients had multiple micro/macronutrient deficiencies, eg, magnesium deficiency. ${ }^{21}$ The risk of NAFLD was also linked with dietary components of the diet, eg, dietary insulinemic potential. ${ }^{22}$ NAFLD and T2DM interacted in a "cause or consequence" fashion such that the presence of one entity increases the predisposition and/or worsens the complications for the other disease. ${ }^{23-27}$ Based on the controversy over the relationship between vitamin $\mathrm{D}$ and NAFLD in patients with T2DM, so we aimed to explore the relationship between $25(\mathrm{OH}) \mathrm{D}$ and NAFLD and to investigate whether there was any difference between different populations.

\section{Methods}

\section{Patients and Study Design}

The study was conducted following the Declaration of Helsinki and was approved by the Ethics Committee of Hebei General Hospital. Our study included 434 patients diagnosed with T2DM who were hospitalized in Hebei General Hospital of Shijiazhuang in China. All subjects had signed written informed consent. Studies were independently assessed by the two researchers and evaluated against inclusion and exclusion criteria by consensus.

\section{Inclusion Criteria}

1) Age $\geq 18$ years. 2) The clinical diagnosis of T2DM was established according to the following criteria: 1) Patients who were under treatment with either oral antidiabetic agents or insulin, and who gave a history of diabetes, also were considered to have T2DM. 2) T2DM conformed to 1999 WHO Diagnostic Criteria: typical symptoms of diabetes (such as polyphagia, polydipsia, polyuria and weight loss) and random blood glucose $\geq 11.1 \mathrm{mmol} / 1$ or fasting blood glucose (FPG) $\geq 7.0 \mathrm{mmol} / 1$ or glucose tolerance test two-hour blood glucose $>11.1 \mathrm{mmol} / 1$. Patients without typical symptoms of diabetes should be rechecked another day. 3) Fatty liver was diagnosed by the findings of ultrasound performed by trained technicians. The participants with liver contrast and liver brightness among the four known criteria (hepatorenal echo contrast, liver brightness, deep attenuation, and vascular blurring) were diagnosed as having fatty liver. ${ }^{28}$

\section{Exclusion Criteria}

1) Patients with type 1 diabetes mellitus, gestational diabetes and other particular types of diabetes were excluded. 2) Patients were excluded if they had a history of myocardial infarction, cerebral haemorrhage, severe dysfunction of liver and kidney, recent acute infection and stress within the last 3 months before enrollment. 3) IPatients were excluded if they had taken calcium, vitamin D supplements or any drugs affecting vitamin D metabolism in the past 6 months.4) Any participants currently using drugs that can increase NAFLD risk, such as amiodarone, corticosteroids, etc. 5) Patients were also excluded if they had a clear drinking history ( $\geq 30$ grams/day in men and $\geq 20$ grams/day in women). Subjects with positive for hepatitis B surface antigen (HBsAg) or anti-hepatitis C virus (HCV) antibody, autoimmune hepatitis, Wilson disease, primary biliary cirrhosis, primary sclerosing cholangitis, liver metabolic disorders, drug-induced hepatitis, hemochromatosis, malignancy, osteoporosis, thyroid disease or kidney disease were also excluded. 


\section{Data Collection}

A questionnaire was utilized to collect basic information from T2DM patients, including age, sex, course of diabetes, history of hypertension, and medication history. Height $(\mathrm{m})$ and weight $(\mathrm{kg})$ were measured to calculate body mass index [BMI = weight $(\mathrm{kg}) /$ height $\left.\left(\mathrm{m}^{2}\right)\right]$. Venous blood samples were drawn from all subjects after an overnight fast of 8 hours. Blood measurements included glycosylated haemoglobin (HbA1c), FPG, albumin, alanine aminotransferase (ALT), aspartate aminotransferase (AST), $\gamma$-glutamyl transferase (GGT), total cholesterol (TC), triglyceride (TG), high-density lipoprotein cholesterol (HDL), low-density lipoprotein cholesterol (LDL), very-low-density lipoprotein cholesterol (VLDL), 25(OH) D. The data and results will be entered into spreadsheets and cross-checked by two researchers. Serum 25(OH)D was determined by electrochemiluminescence immunoassays (ECLA) using Roche commercial kits (COBAS e 601, Roche, Germany).

\section{Statistical Analysis}

Statistical analyses were performed using SPSS software, version 25.0. Continuous variables were expressed as the mean \pm standard deviation (SD) if normally distributed and as medians and interquartile range (P25, P75) if not normally distributed. Independent group $t$-test was used for a continuous variable when the data conformed to normal distribution; otherwise, the Kruskal-Wallis test was applied. The enumeration data were expressed in percentage $[\mathrm{n} /(\%)]$, and the Chisquare test tested the comparison between the groups. Descriptive statistics were calculated and compared between T2DM patients with and without NAFLD or vitamin D deficiency. The variables with a p-value level of less than 0.1 were entered into the multiple logistic regression model. Differences were considered statistically significant for $p$ values $<0.05$. Taking $\alpha=0.05, \beta=0.10$, and bilateral testing, the PASS software (version 15.0) was used to calculate the sample size in this study.

\section{Results}

\section{Comparison of Clinical Characteristics Between T2DM Patients with and without NAFLD}

By comparing the clinical parameters of T2DM patients with NAFLD and those without NAFLD, there were no statistically differences in sex, the proportion of hypertension, the level of FPG and HbAlc between the two groups. Patients with NAFLD had a significantly shorter duration $(p=0.049)$ and younger age $(p=0.004)$ than patients without NAFLD. The levels of BMI $(p<0.001)$, albumin $(p=0.030)$, ALT $(p<0.001)$, AST $(p<0.001)$, GGT $(p<0.001)$, TC $(p=0.002)$, TG $(p<0.001)$, LDL $(p=0.004)$, VLDL $(p<0.001)$ were higher among T2DM patients who had NAFLD compared to those without NAFLD. Patients with combined T2DM and NAFLD had decreased HDL levels compared with T2DM patients (Table 1).

\section{Comparison of Clinical Characteristics in T2DM Patients with VDD and VDND Groups}

Patients were divided into two groups according to their 25(OH)D levels: vitamin D-deficient group (VDD) $(<20 \mathrm{ng} / \mathrm{mL}$ ), and vitamin D non-deficient group (VDND) $(>20 \mathrm{ng} / \mathrm{mL}$ ), with 296, 138 patients in each group, respectively. In the VDD group, BMI ( $\mathrm{p}=0.03)$, HbA1c $(p=0.002)$, TG $(p<0.001)$, VLDL $(p=0.009)$ levels and female proportion $(p<0.001)$ were significantly higher compared with the VDND group, while the levels of albumin were lower than T2DM patients in the VDND group. However, there was no significant difference in age, T2DM course, ALT, AST, GGT, TC, HDL, LDL, FPG and hypertension proportion between the VDD and VDND groups (Table 2).

\section{Comparison of 25(OH)D Levels and Prevalence of NAFLD in T2DM Patients with Different Levels of BMI}

Patients who underwent combined NAFLD still conferred a statistically significant lower level of 25(OH)D [15.89 $(12.33,19.94)$ vs $18.27(14.05,24.63), p<0.001]$ (Figure 1A) and a higher proportion of patients with vitamin 
Table I Characteristics of Patients with and without NAFLD Included in This Study

\begin{tabular}{|c|c|c|c|c|}
\hline & All Participants & Without NAFLD & With NAFLD & $P$ value \\
\hline n (\%) & 434 & 200 & 234 & - \\
\hline Age & $58.74 \pm 12.92$ & $60.79 \pm 11.99$ & $56.98 \pm 13.45$ & 0.004 \\
\hline $\operatorname{Sex}(F, \%)$ & $184(42.4 \%)$ & 85 (42.5\%) & $99(42.3 \%)$ & 0.968 \\
\hline Hypertension (n, \%) & 217 (50\%) & 94 (47\%) & $123(52.6 \%)$ & 0.248 \\
\hline DM course (years) & $9.87(3,15)$ & $10.00(3.00,16.00)$ & $8.00(2.00,15.00)$ & 0.049 \\
\hline BMI $\left(\mathrm{kg} / \mathrm{m}^{2}\right)$ & $25.72 \pm 3.95$ & $23.7 I \pm 3.27$ & $27.44 \pm 3.65$ & $<0.001$ \\
\hline Albumin (g/L) & $40.18(37.68,42.93)$ & $39.85(37.60,42.60)$ & $40.80(37.78,43.20)$ & 0.030 \\
\hline ALT (U/L) & $24.42(13.70,28.50)$ & $15.70(12.53,22.58)$ & $21.20(15.50,34.45)$ & $<0.001$ \\
\hline AST (U/L) & $22.50(15.70,24.10)$ & $17.90(14.63,21.88)$ & $20.00(16.70,26.80)$ & $<0.001$ \\
\hline GGT (U/L) & $38.20(17.15,38.65)$ & $20.60(14.00,29.63)$ & $30.00(19.60,46.00)$ & $<0.001$ \\
\hline HbAlc (\%) & $8.97(7.18,10.50)$ & $8.40(6.90,10.40)$ & $8.80(7.40,10.50)$ & 0.089 \\
\hline FPG $(\mathrm{mmol} / \mathrm{l})$ & $8.87(6.29,11.15)$ & $7.62(6.12,10.54)$ & $8.38(6.42,11.54)$ & 0.163 \\
\hline $\mathrm{TC}(\mathrm{mmol} / \mathrm{L})$ & $4.77(3.88,5.42)$ & $4.45(3.76,5.18)$ & $4.77(4.01,5.59)$ & 0.002 \\
\hline TG (mmol/L) & $2.05(1.03,2.33)$ & $1.16(0.87,1.75)$ & $1.86(1.26,2.7 I)$ & $<0.001$ \\
\hline $\mathrm{HDL}(\mathrm{mmol} / \mathrm{L})$ & $1.05(0.84,1.18)$ & $1.04(0.89,1.24)$ & $0.97(0.83,1.15)$ & 0.002 \\
\hline LDL (mmol/L) & $3.11(2.46,3.6 I)$ & $2.90(2.33,3.48)$ & $3.22(2.59,3.72)$ & 0.004 \\
\hline VLDL (mmol/l) & $0.5 I(0.35,0.69)$ & $0.43(0.28,0.58)$ & $0.57(0.40,0.80)$ & $<0.001$ \\
\hline $25(\mathrm{OH}) \mathrm{D}(\mathrm{mmol} / \mathrm{l})$ & $17.04(12.99,21.63)$ & I8.27 (14.05, 24.63) & $15.89(12.33,19.94)$ & $<0.001$ \\
\hline Vitamin D deficiency (\%) & 296 (68.2\%) & II 8 (59\%) & 178 (76.1\%) & $<0.001$ \\
\hline
\end{tabular}

D deficiency $(76.1 \%$ vs $59 \%, P<0.001)$ compared with T2DM patients without NAFLD (Table 1). NAFLD prevalence in T2DM patients with $25(\mathrm{OH}) \mathrm{D}$ deficiency was higher than in those without $25(\mathrm{OH}) \mathrm{D}$ deficiency, and the difference was statistically significant (60.1\% vs $40.6 \%, P<0.001)$ (Figure $2 \mathrm{~A})$.

BMI was categorized as BMI $\leq 23 \mathrm{~kg} / \mathrm{m}^{2}$ and BMI $\geq 23 \mathrm{~kg} / \mathrm{m}^{2}$. We also carried out separate analyses for these two subgroups. In T2DM patients with BMI $>23 \mathrm{~kg} / \mathrm{m}^{2}$, the level of $25(\mathrm{OH}) \mathrm{D}$ was significantly lower in T2DM patients with NAFLD compared with those without NAFLD $[15.80(12.04,19.73)$ vs $17.72(13.36,23.57), P=0.002]$ (Figure 1B). The NAFLD prevalence in patients with $25(\mathrm{OH}) \mathrm{D}$ deficiency was higher than in those without $25(\mathrm{OH}) \mathrm{D}$ deficiency, and the difference was statistically significant $(68.4 \%$ vs $51 \%, P=0.002)$ in T2DM patients with BMI $>23 \mathrm{~kg} / \mathrm{m}^{2}$ (Figure $2 \mathrm{~B}$ ). However, no difference was observed in the levels of $25(\mathrm{OH})$ D between NAFLD and non-NAFLD groups in T2DM patients with BMI $\leq 23 \mathrm{~kg} / \mathrm{m}^{2}$ (Figure $1 \mathrm{C}$ ). There was no difference in the prevalence of NAFLD between VDND and VDD groups in T2DM patients with BMI $\leq 23 \mathrm{~kg} / \mathrm{m}^{2}$ (Figure $2 \mathrm{C}$ ).

\section{Spearman Correlation of 25(OH)D with Potential NAFLD Factors}

Correlation analysis demonstrated that $25(\mathrm{OH}) \mathrm{D}$ level was positively correlated with albumin $(r=0.214, \mathrm{p}<0.001)$ and negatively correlated with BMI $(r=-0.138, p=0.004)$, FPG $(r=-0.112, p=0.019), \mathrm{HbA1c}(r=-0.190, p<0.001)$, TG $(r=$ $-0.231, p<0.001)$, LDL $(r=-0.103, p=0.032)$, VLDL $(r=-0.151, p=0.002)$ in all study subjects. Figure 3 demonstrates the results of Spearman correlation analysis. 
Table 2 Characteristics of Patients with and without Vitamin D Deficiency Included in This Study

\begin{tabular}{|c|c|c|c|}
\hline & VDND & VDD & $P$ value \\
\hline$n$ & 138 & 296 & - \\
\hline Age & $58.1756 \pm 12.91658$ & $58.9965 \pm 12.94043$ & 0.547 \\
\hline $\operatorname{Sex}(F, \%)$ & 39 (28.3\%) & 145 (49\%) & $<0.001$ \\
\hline Hypertension (n, \%) & $60(43.5 \%)$ & I57 (53\%) & 0.064 \\
\hline NAFLD & 56 (40.6\%) & $178(60.1 \%)$ & $<0.001$ \\
\hline DM course (years) & $7.5(3,15)$ & $9(3,15)$ & 0.834 \\
\hline BMI $\left(\mathrm{kg} / \mathrm{m}^{2}\right)$ & $25.345(22.865,26.8975)$ & $25.755(23.7025,28.0975)$ & 0.03 \\
\hline Albumin (g/L) & $41.15(38.3,43.8)$ & $40.1(37.025,42.6)$ & 0.001 \\
\hline ALT (U/L) & $19.85(13.925,29.8)$ & $18.4(13.7,27.5)$ & 0.66 \\
\hline AST (U/L) & $19.1(15.7,24.6)$ & 19.2(15.7, 23.9) & 0.988 \\
\hline GGT (U/L) & $24.65(16.75,37.6)$ & $25.9(17.7,38.7)$ & 0.383 \\
\hline $\mathrm{HbAlc}(\%)$ & $8.2(6.875,9.7)$ & $8.9(7.4,10.5)$ & 0.002 \\
\hline FPG (mmol/l) & $7.55(5.84,10.62)$ & $8.225(6.425,11.18)$ & 0.079 \\
\hline TC (mmol/L) & $4.555(3.78,5.2425)$ & $4.665(3.8925,5.505)$ & 0.072 \\
\hline TG (mmol/L) & $1.25(0.9175,1.855)$ & $1.655(1.0825,2.4625)$ & $<0.001$ \\
\hline HDL-C (mmol/L) & I.0I(0.88, I.I825) & $\mathrm{I}(0.83, \mathrm{I} .1875)$ & 0.345 \\
\hline LDL-C (mmol/L) & $2.98(2.325,3.4525)$ & $3.09(2.47,3.72)$ & 0.071 \\
\hline VLDL (mmol/L) & $0.46(0.3075,0.6 I)$ & $0.53(0.37,0.72)$ & 0.009 \\
\hline
\end{tabular}

\section{Multivariate Logistic Regression Analyses of NAFLD and Associated Factors}

Multiple logistic regression analysis was employed to examine whether vitamin D deficiency was independently and significantly associated with the presence of NAFLD in T2DM patients. For all T2DM patients, vitamin D deficiency was
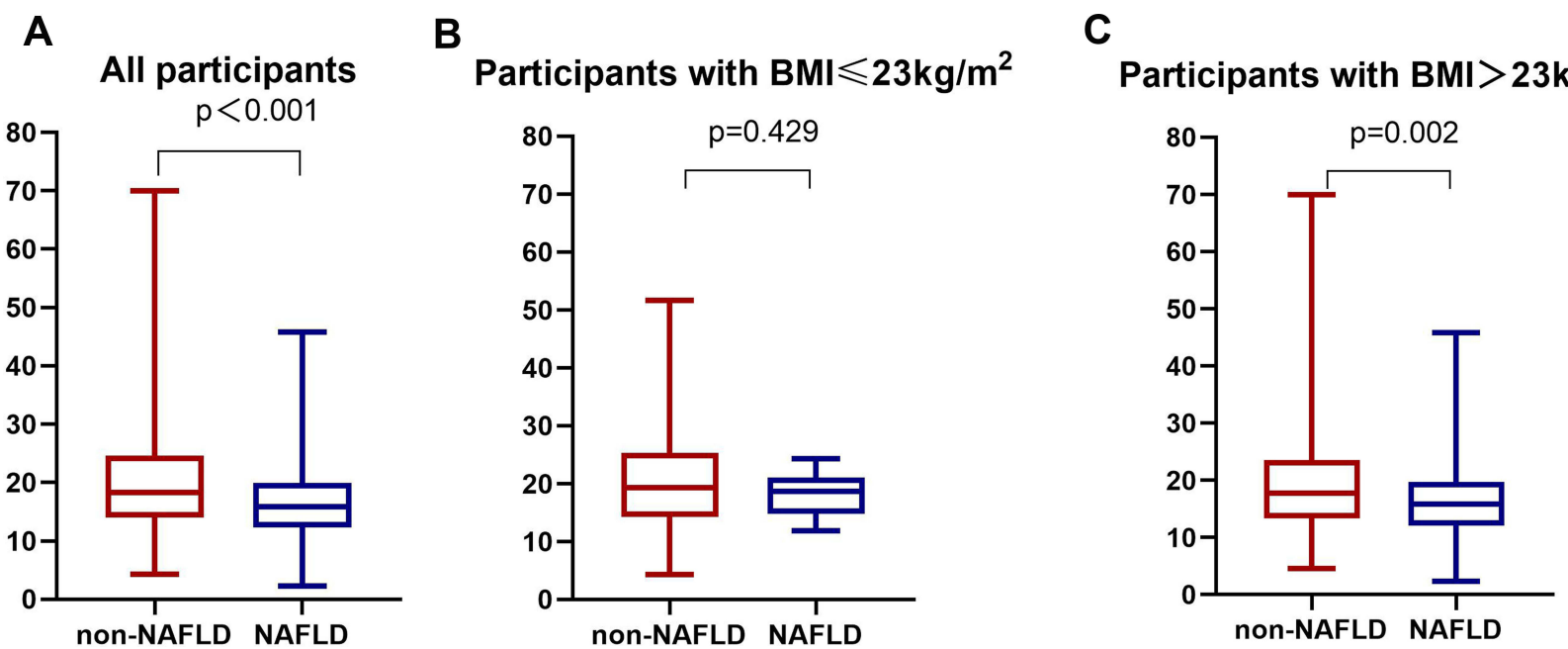

Figure I Comparison of 25(OH)D between the NAFLD group and the non-NAFLD group in participants with T2DM: (A) all participants; (B) participants with BMI >23kg/ $\mathrm{m}^{2}$; (C) participants with BMI $\leq 23 \mathrm{~kg} / \mathrm{m}^{2}$. 
A

All participants

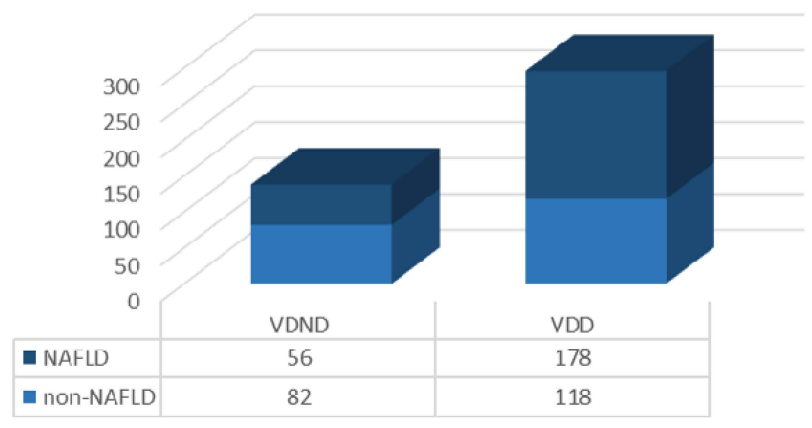

B

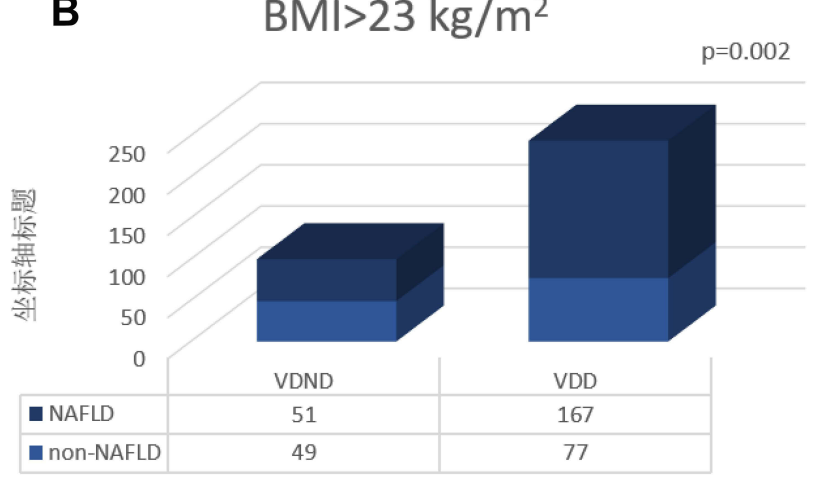

\section{C $\quad B M l \leq 23 \mathrm{~kg} / \mathrm{m} 2$}

$p=0.327$

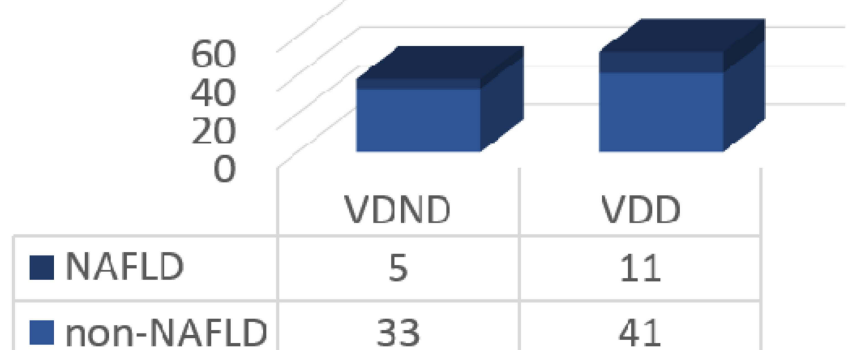

Figure 2 Comparison of the prevalence of NAFLD in T2DM patients with and without vitamin D deficiency: (A) all participants; (B) participants with BMI >23kg/ $\mathrm{m}^{2}$; (C) participants with $\mathrm{BMI} \leq 23 \mathrm{~kg} / \mathrm{m}^{2}$.

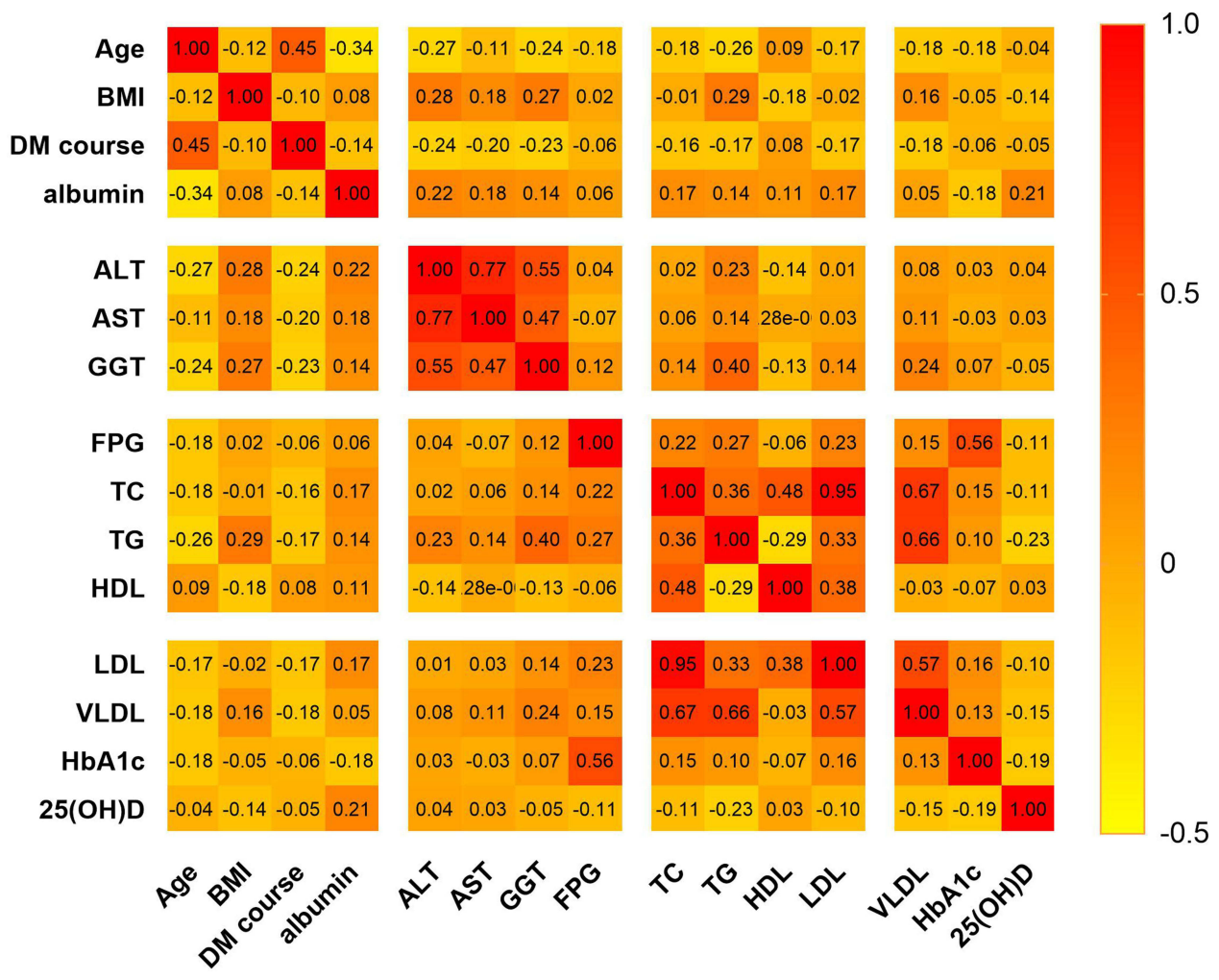

Figure 3 Spearman correlation analysis of $25(\mathrm{OH}) \mathrm{D}$ with potential risk factors for NAFLD. 
Table 3 Logistic Regression Analysis of Vitamin D Deficiency for NAFLD in Patients with T2DM

\begin{tabular}{|l|l|l|l|l|l|l|}
\hline & B & Std. Error & Wald & P & OR & 95\% CI (OR) \\
\hline Model I & 0.843 & 0.262 & 10.4 & 0.001 & 2.324 & $(1.392,3.88)$ \\
\hline Model 2 & 0.863 & 0.269 & 10.269 & 0.001 & 2.369 & $(1.398,4.016)$ \\
\hline Model 3 & 0.715 & 0.286 & 6.246 & 0.012 & 2.045 & $(1.167,3.583)$ \\
\hline
\end{tabular}

Notes: Model I: adjusted for sex, age, BMI, hypertension; Model 2: adjusted for sex, age, BMI, hypertension, DM course, albumin, ALT, AST, GGT; Model 3: adjusted for sex, age, BMI, hypertension, DM course, albumin, ALT, AST, GGT, FPG, TC, TG, HDL, LDL, VLDL, HbAlc.

Table 4 Logistic Regression Analysis of Vitamin D Deficiency for NAFLD in T2DM Patients with BMI $\leq 23 \mathrm{~kg} / \mathrm{m}^{2}$

\begin{tabular}{|l|l|l|l|l|l|l|}
\hline & B & Std. Error & Wald & P & OR & 95\% CI \\
\hline Model I & 0.862 & 0.696 & 1.534 & 0.216 & 2.367 & $(0.605,9.261)$ \\
\hline Model 2 & 0.245 & 0.782 & 0.098 & 0.754 & 1.278 & $(0.276,5.921)$ \\
\hline Model 3 & -0.21 & 1.03 & 0.042 & 0.838 & 0.811 & $(0.108,6.097)$ \\
\hline
\end{tabular}

Notes: Model I: adjusted for sex, age, BMI, hypertension; Model 2: adjusted for sex, age, BMI, hypertension, DM course, albumin, ALT, AST, GGT; Model 3: adjusted for sex, age, BMI, hypertension, DM course, albumin, ALT, AST, GGT, FPG, TC, TG, HDL, LDL, HbAlc.

significantly associated with a higher prevalence of NAFLD. Specifically, T2DM patients with vitamin D deficiency had a $2.045(95 \%$ CI: 1.167, 3.583) times higher risk of developing NAFLD than those without vitamin D deficiency (Table 3).

Vitamin D deficiency was not associated with high NAFLD preference in T2DM patients with BMI $\leq 23 \mathrm{~kg} / \mathrm{m}^{2},(P=$ 0.216 for model $1, P=0.754$ for model 2, and $P=0.838$ for model 3) (Table 4). Vitamin D deficiency was associated with high NAFLD preference in T2DM patients with BMI $>23 \mathrm{~kg} / \mathrm{m}^{2}$, regardless of whether an unadjusted or adjusted model was used ( $P=0.004$ for model $1, P=0.003$ for model 2, and $P=0.016$ for model 3) (Table 5).

\section{Subgroup Analysis}

To further investigate the impact of other risk factors on the correlation of vitamin D deficiency with NAFLD risk in T2DM patients with BMI $>23 \mathrm{~kg} / \mathrm{m}^{2}$, subgroup analyses were carried according to sex, age, history of hypertension, TG, HDL and HbA1c. Table 6 has summarized the results of subgroup analysis results. The significant correlation between vitamin D deficiency and NAFLD was found in participants with age $\leq 65$ years, without hypertension, $\mathrm{TG}<1.7 \mathrm{mmol} / 1$, $\mathrm{HDL} \geq 1 \mathrm{mmol} / 1$ in men, $\geq 1.3 \mathrm{mmol} / 1$ in women, HBA1C $\leq 7 \%$, or females.

\section{Discussion}

The results of this study revealed that the 25(OH)D level in patients with T2DM complicated by NAFLD was significantly lower than that in patients with T2DM only. It was consistent with the result of Xiu et al. ${ }^{29}$ Recently, it was shown that bariatric surgery could also influence vitamin D levels in obese patients. ${ }^{30}$ No patients in this study underwent bariatric surgery. A meta-analysis of 10 RCTs (randomized controlled trials) showed that Sodium-Glucose Transporter 2 (SGLT2) inhibitors could remarkably reduce hepatic enzymes and hepatic fat. ${ }^{31}$ Another meta-analysis

Table 5 Logistic Regression Analysis of Vitamin D Deficiency for NAFLD in T2DM Patients with BMI $>23 \mathrm{~kg} / \mathrm{m}^{2}$

\begin{tabular}{|l|l|l|l|l|l|l|}
\hline & B & Std. Error & Wald & P & OR & 95\% CI (OR) \\
\hline Model I & 0.809 & 0.282 & 8.215 & 0.004 & 2.246 & $(1.292,3.906)$ \\
\hline Model 2 & 0.849 & 0.29 & 8.595 & 0.003 & 2.338 & $(1.325,4.126)$ \\
\hline Model 3 & 0.747 & 0.312 & 5.753 & 0.016 & 2.111 & $(1.146,3.888)$ \\
\hline
\end{tabular}

Notes: Model I: adjusted for sex, age, BMI, hypertension; Model 2: adjusted for sex, age, BMI, hypertension, DM course, albumin, ALT, AST, GGT; Model 3: adjusted for sex, age, BMI, hypertension, DM course, albumin, ALT, AST, GGT, FPG, TC, TG, HDL, LDL, VLDL, HbAlc. 
Table 6 Effect of Vitamin D Deficiency on NAFLD Risk Stratified by Subgroups

\begin{tabular}{|c|c|c|c|}
\hline Characteristics & No. of Participants & OR(95\% Cl) & P-value \\
\hline \multicolumn{4}{|l|}{ Age (years) } \\
\hline$\leq 65$ & 306 & $2.374(I .144,4.923)$ & 0.02 \\
\hline$>65$ & 128 & I.184(0.267, 5.248) & 0.824 \\
\hline \multicolumn{4}{|l|}{ Sex } \\
\hline Males & 250 & $1.345(0.633,2.857)$ & 0.44 \\
\hline Females & 184 & 6.64I(I.57I, 28.072) & 0.01 \\
\hline \multicolumn{4}{|l|}{ Hypertension } \\
\hline No & 217 & $3.275(1.225,8.759)$ & 0.018 \\
\hline Yes & 217 & $|.56|(0.66 \mid, 3.687)$ & 0.31 \\
\hline \multicolumn{4}{|l|}{ HBAIC (\%) } \\
\hline$\leq 9$ & 246 & $1.986(0.899,4.385)$ & 0.09 \\
\hline$>9$ & 188 & $2.007(0.587,6.86)$ & 0.587 \\
\hline \multicolumn{4}{|l|}{ TG (mmol/l) } \\
\hline$\geq 1.7$ & 187 & $0.673(0.183,2.477)$ & 0.551 \\
\hline$<1.7$ & 247 & $2.813(1.297,6.101)$ & 0.009 \\
\hline \multicolumn{4}{|l|}{$\mathrm{HDL}(\mathrm{mmol} / \mathrm{l})$} \\
\hline$\geq 1$ in men, $\geq 1.3$ in women & 149 & $4.947(1.204,20.315)$ & 0.027 \\
\hline$<1$ in men, $<1.3$ in women & 285 & $2.04(0.905,4.598)$ & 0.085 \\
\hline
\end{tabular}

found that pioglitazone could significantly improve the histological performance of the liver and insulin sensitivity. ${ }^{32}$ Moreover, this study demonstrated that none of the T2DM patients used SGLT2 inhibitors or pioglitazone.

Our results indicated that vitamin D deficiency was highly prevalent among T2DM patients with NAFLD. It was demonstrated that the relation between vitamin D deficiency and NAFLD was bidirectional. On the one hand, vitamin D (either synthesized in the skin - cholecalciferol - or obtained from dietary - ergocalciferol) was metabolized within the liver to $25(\mathrm{OH}) \mathrm{D}$ and within the kidneys to the biologically active $(1,25$ - dihydroxy vitamin $\mathrm{D}) .{ }^{33}$ On the other hand, insulin resistance was at the root of both T2DM and NAFLD, and vitamin D may significantly impact insulin sensibilization through its anti-inflammatory effects and its role in regulating insulin secretion. ${ }^{34}$

Because obesity was a confounding factor, ${ }^{35}$ we measured BMI, a surrogate measure of obesity, and it had also mainly been demonstrated. ${ }^{36}$ Several recent studies recommend that BMI may be best maintained within $23 \mathrm{~kg} / \mathrm{m}^{2} .{ }^{37-41}$ Therefore, we performed multiple regression analysis in all T2DM subjects and T2DM patients with BMI $>23 \mathrm{~kg} / \mathrm{m}^{2}$ and BMI $\leq 23 \mathrm{~kg} / \mathrm{m}^{2}$, respectively. We observed that vitamin D deficiency was an independent risk factor for NAFLD in T2DM patients, especially in those with BMI $>23 \mathrm{~kg} / \mathrm{m}^{2}$.

The explanation for why obese and lean individuals have inconsistent correlations between vitamin D and NAFLD remains unclear, although several possibilities exist. ${ }^{42}$ First, obesity could be a contributing factor to low vitamin D levels. Second, patients with vitamin D deficiency have higher serum levels of proinflammatory cytokines, which promote NAFLD development. Third, under vitamin D deficiency conditions, the flow of free fatty acids (FFAs) in the blood increases, and fat deposition into hepatocytes is accelerated, contributing to the progress of NAFLD.

It was recognised that a strong association between a low level of $25(\mathrm{OH}) \mathrm{D}$ and NAFLD existed in patients with T2DM. ${ }^{43}$ Another study conducted in Korea has also reported similar results indicating a negative association between 
25(OH)D and NAFLD after adjustment for potential confounders. ${ }^{44}$ Recently, Nelson et a ${ }^{45}$ reported that $25(\mathrm{OH}) \mathrm{D}$ deficiency was commonly associated with the increased risk of NAFLD diagnosed by liver biopsy. In a meta-analysis by Eliades et $\mathrm{al}^{20}{ }^{20} 25(\mathrm{OH}) \mathrm{D}$ level was lower in patients diagnosed with NAFLD. In addition, several studies have demonstrated that vitamin D status was associated with dyslipidemia, inflammation, insulin resistance, oxidative stress and obesity. ${ }^{11,46-49}$ Vitamin D levels can be modulated by consuming other drugs/supplements, eg, omega-3 fatty acids. ${ }^{50}$ Thus, there have been recommendations that taking vitamin supplements may potentially contribute to NAFLD treatment. However, the results are inconsistent. Findings by Talaei et $\mathrm{al}^{51}$ suggested that a 2-month course of vitamin D supplementation improved insulin resistance in patients with T2DM. A study by Papapostoli et $\mathrm{al}^{52}$ had demonstrated that vitamin D supplementation reduced the severity of hepatic steatosis in patients with NAFLD. The results of Barchetta et $\mathrm{al}^{7}$ revealed that hepatic steatosis was not able to improve after 24 weeks of supplementation of vitamin D. As pointed out by Heaney, ${ }^{53}$ a central bias behind the inconsistent results from trials on the effects of vitamin D supplementation may be represented by the well-known physiology of nutrients, which do not show the dose-response relation observed for most drugs. Results from a recent meta-analysis that included 16 RCTs indicated that Vitamin D supplementation could be considered an effective strategy in managing patients with NAFLD. ${ }^{54}$ Patients with NAFLD were also prone to develop liver fibrosis and even liver cancer if they were associated with vitamin D deficiency. ${ }^{55,56}$

Vitamin D deficiency may play a role in the pathogenesis of NAFLD by decreasing the expression of the glucose transporter, reducing hepatic glucose output, and stimulating intrahepatic lipid synthesis. ${ }^{57}$ Thus, a low serum vitamin D level was potentially prone to intrahepatic lipid accumulation, leading to NAFLD. The first "two hits" theory of NAFLD pathogenesis mainly involved insulin resistance and excessive free fatty acids in the circulation. ${ }^{58}$ Vitamin D decreased insulin resistance induced by free fatty acids in peripheral tissues and hepatocytes. ${ }^{59}$ In addition, Thais et al's study ${ }^{60}$ also showed insulin resistance could be improved in vitamin D-supplemented animals. Moreover, they proved vitamin D deficiency had a particularly adverse impact on metabolism, hepatic steatosis, and changes caused by hepatic stellate cell activation (liver fibrosis, $\beta$-oxidation, lipogenesis, and liver inflammation). Animals appeared to improve all these data after vitamin D supplementation.

Oxidative stress was considered the second hit of the "two hits" theory of NAFLD pathogenesis. Some studies have demonstrated higher oxidative stress biomarkers in people with a low 25(OH)D level. Intake of vitamin D also decreased oxidative stress. ${ }^{61,62}$ Indeed, Eftekhari et $\mathrm{al}^{63}$ found that the intake of calcitriol $(1,25(\mathrm{OH}) 2 \mathrm{D} 3)$ significantly reduced TC, LDL, TG, and malondialdehyde levels in patients with T2DM. They concluded that 1, 25(OH)2D3 could reduce oxidative stress and improve lipid profiles. Nakano et $\mathrm{al}^{64}$ recently found that sunlight therapy ameliorated hepatocyte inflammation and fibrosis by regulating lipid transfer/metabolic. Inflammation could also lead to the development and progression of NAFLD. ${ }^{65}$ Vitamin D was also known to exhibit anti-inflammatory effects on macrophages. ${ }^{66}$ The study also demonstrated that vitamin D deficiency was associated with impaired T lymphocyte immune function and increased inflammation through elevated levels of proinflammatory cytokines. ${ }^{67}$ A potentially novel insight was provided into the interactions of the liver and intestine in the progression and pathogenesis of NAFLD. ${ }^{68}$ Vitamin D deficiency facilitated non-alcoholic steatohepatitis progression by impaired enterohepatic circulation in animal models. ${ }^{69}$ Hamed et al showed that the NAFLD patients with T2DM were characterized by different gut compositions, ${ }^{70}$ which supported the role of gut microbiota in the pathogenesis of T2DM and NAFLD. The findings from this study suggest intake of vitamin D should be emphasized in order to prevent NAFLD in obese T2DM populations.

The present study had a few limitations. Firstly, a causal relationship cannot be established due to the cross-sectional study design. Further studies are required to evaluate the causative relationships between 25(OH)D and NAFLD, the effect of vitamin D supplementation on NAFLD, and patients with T2DM. Secondly, the diagnosis of NAFLD was based on ultrasonography and the exclusion of other known causes of liver disease but was not confirmed by liver biopsy. Third, the BMI classification criteria applied in this study are limited to Asian people, so our findings may not be generalizable to other ethnic populations.

\section{Conclusion}

This study suggests that T2DM peope with BMI $>23 \mathrm{~kg} / \mathrm{m}^{2}$ were more susceptible to NAFLD by vitamin D deficiency and that it is necessary to maintain optimal serum vitamin D levels in this population. 


\section{Data Sharing Statement}

The original data can be available by email request at any time (Yuling Xing:xingy195@163.com).

\section{Funding}

There is no funding to report.

\section{Disclosure}

The authors declare that they have no conflicts of interest.

\section{References}

1. Farrell GC, Larter CZ. Nonalcoholic fatty liver disease: from steatosis to cirrhosis. Hepatology. 2006;43(2 Suppl 1):S99-s112. doi:10.1002/ hep. 20973

2. Younossi ZM, Golabi P, de Avila L, et al. The global epidemiology of NAFLD and NASH in patients with type 2 diabetes: a systematic review and meta-analysis. J Hepatol. 2019;71(4):793-801. doi:10.1016/j.jhep.2019.06.021

3. Amiri Dash Atan N, Koushki M, Motedayen M, et al. Type 2 diabetes mellitus and non-alcoholic fatty liver disease: a systematic review and meta-analysis. Gastroenterol Hepatol Bed Bench. 2017;10(Supp11):S1-s7.

4. Dai W, Ye L, Liu A, et al. Prevalence of nonalcoholic fatty liver disease in patients with type 2 diabetes mellitus: a meta-analysis. Medicine. 2017;96(39):e8179. doi:10.1097/MD.0000000000008179

5. Chalasani N, Younossi Z, Lavine JE, et al. The diagnosis and management of non-alcoholic fatty liver disease: practice guideline by the American Association for the Study of Liver Diseases, American College of Gastroenterology, and the American Gastroenterological Association. Am J Gastroenterol. 2012;107(6):811-826. doi:10.1038/ajg.2012.128

6. Mikolasevic I, Milic S, Turk Wensveen T, et al. Nonalcoholic fatty liver disease - A multisystem disease? World J Gastroenterol. 2016;22 (43):9488-9505. doi:10.3748/wjg.v22.i43.9488

7. Barchetta I, Del Ben M, Angelico F, et al. No effects of oral vitamin D supplementation on non-alcoholic fatty liver disease in patients with type 2 diabetes: a randomized, double-blind, placebo-controlled trial. BMC Med. 2016;14:92. doi:10.1186/s12916-016-0638-y

8. Christakos S, Dhawan P, Verstuyf A, Verlinden L, Carmeliet G. Vitamin D: metabolism, molecular mechanism of action, and pleiotropic effects. Physiol Rev. 2016;96(1):365-408. doi:10.1152/physrev.00014.2015

9. Tsuprykov O, Chen X, Hocher CF, Skoblo R, Lianghong Y, Hocher B. Why should we measure free 25(OH) vitamin D? J Steroid Biochem Mol Biol. 2018;180:87-104. doi:10.1016/j.jsbmb.2017.11.014

10. Pham H, Waterhouse M, Baxter C, et al. The effect of vitamin D supplementation on acute respiratory tract infection in older Australian adults: an analysis of data from the D-Health Trial. Lancet Diabetes Endocrinol. 2021;9(2):69-81. doi:10.1016/S2213-8587(20)30380-6

11. Gagnon C, Lu ZX, Magliano DJ, et al. Low serum 25-hydroxyvitamin D is associated with increased risk of the development of the metabolic syndrome at five years: results from a national, population-based prospective study (The Australian Diabetes, Obesity and Lifestyle Study: ausDiab). J Clin Endocrinol Metab. 2012;97(6):1953-1961. doi:10.1210/jc.2011-3187

12. Lips P, Eekhoff M, van Schoor N, et al. Vitamin D and type 2 diabetes. J Steroid Biochem Mol Biol. 2017;173:280-285. doi:10.1016/j. jsbmb.2016.11.021

13. Sacerdote A, Dave P, Lokshin V, Bahtiyar G. Type 2 diabetes mellitus, insulin resistance, and Vitamin D. Curr Diab Rep. $2019 ; 19(10): 101$. doi:10.1007/s11892-019-1201-y

14. Rhee EJ, Kim MK, Park SE, et al. High serum vitamin D levels reduce the risk for nonalcoholic fatty liver disease in healthy men independent of metabolic syndrome. Endocr J. 2013;60(6):743-752. doi:10.1507/endocrj.EJ12-0387

15. Manson JE, Cook NR, Lee IM, et al. Vitamin D supplements and prevention of cancer and cardiovascular disease. N Engl J Med. 2019;380 (1):33-44. doi:10.1056/NEJMoa1809944

16. Pollock RD, Duggal NA, Lazarus NR, Lord JM, Harridge SDR. Cardiorespiratory fitness not sedentary time or physical activity is associated with cardiometabolic risk in active older adults. Scand J Med Sci Sports. 2018;28(6):1653-1660. doi:10.1111/sms.13071

17. Holick MF. The vitamin D deficiency pandemic: approaches for diagnosis, treatment and prevention. Rev Endocr Metab Disord. 2017;18 (2):153-165. doi:10.1007/s11154-017-9424-1

18. Jiang Z, Pu R, Li N, et al. High prevalence of vitamin D deficiency in Asia: a systematic review and meta-analysis. Crit Rev Food Sci Nutr. 2021;1-10. doi:10.1080/10408398.2021.1990850

19. Dasarathy J, Periyalwar P, Allampati S, et al. Hypovitaminosis D is associated with increased whole body fat mass and greater severity of non-alcoholic fatty liver disease. Liver Int. 2014;34(6):e118-127. doi:10.1111/liv.12312

20. Eliades M, Spyrou E, Agrawal N, et al. Meta-analysis: vitamin D and non-alcoholic fatty liver disease. Aliment Pharmacol Ther. 2013;38 (3):246-254. doi:10.1111/apt.12377

21. Găman MA, Dobrică EC, Cozma MA, et al. Crosstalk of magnesium and serum lipids in dyslipidemia and associated disorders: a systematic review. Nutrients. 2021;13(5):1411. doi:10.3390/nu13051411

22. Sohouli MH, Sayyari AA, Lari A, et al. Association of dietary insulinaemic potential and odds of non-alcoholic fatty liver disease among adults: a case-control study. J Hum Nutr Diet. 2021;34(5):901-909. doi:10.1111/jhn.12865

23. Lonardo A, Nascimbeni F, Mantovani A, Targher G. Hypertension, diabetes, atherosclerosis and NASH: cause or consequence? J Hepatol. 2018;68 (2):335-352. doi:10.1016/j.jhep.2017.09.021

24. Loomba R, Abraham M, Unalp A, et al. Association between diabetes, family history of diabetes, and risk of nonalcoholic steatohepatitis and fibrosis. Hepatology. 2012;56(3):943-951. doi:10.1002/hep.25772

25. Li Y, Wang J, Tang Y, et al. Bidirectional association between nonalcoholic fatty liver disease and type 2 diabetes in Chinese population: evidence from the Dongfeng-Tongji cohort study. PLoS One. 2017;12(3):e0174291. doi:10.1371/journal.pone.0174291 
26. Bril F, Cusi K. Nonalcoholic fatty liver disease: the new complication of type 2 diabetes mellitus. Endocrinol Metab Clin North Am. 2016;45 (4):765-781. doi:10.1016/j.ecl.2016.06.005

27. Fukuda T, Hamaguchi M, Kojima T, et al. Transient remission of nonalcoholic fatty liver disease decreases the risk of incident type 2 diabetes mellitus in Japanese men. Eur J Gastroenterol Hepatol. 2016;28(12):1443-1449. doi:10.1097/MEG.0000000000000736

28. Hamaguchi M, Kojima T, Itoh Y, et al. The severity of ultrasonographic findings in nonalcoholic fatty liver disease reflects the metabolic syndrome and visceral fat accumulation. Am J Gastroenterol. 2007;102(12):2708-2715. doi:10.1111/j.1572-0241.2007.01526.x

29. Xiu L, Jiang T, Yao XA, Wen Z. Correlation between 25 Hydroxyvitamin D levels and nonalcoholic fatty liver disease in Chinese patients with type 2 diabetes mellitus: a cross-sectional study. Int J Gen Med. 2021;14:3099-3107. doi:10.2147/IJGM.S319449

30. Bourbour F, Kabir A, Pazouki A, et al. Trends in serum Vitamin D levels within 12 months after One Anastomosis Gastric Bypass (OAGB). Obes Surg. 2021;31(9):3956-3965. doi:10.1007/s11695-021-05434-6

31. Wei Q, Xu X, Guo L, Li J, Li L. Effect of SGLT2 inhibitors on type 2 diabetes mellitus with non-alcoholic fatty liver disease: a meta-analysis of randomized controlled trials. Front Endocrinol. 2021;12:635556. doi:10.3389/fendo.2021.635556

32. Lian J, Fu J. Pioglitazone for NAFLD patients with prediabetes or type 2 diabetes mellitus: a meta-analysis. Front Endocrinol. 2021;12:615409. doi:10.3389/fendo.2021.615409

33. Bjelakovic G, Nikolova D, Bjelakovic M, Gluud C. Vitamin D supplementation for chronic liver diseases in adults. Cochrane Database Syst Rev. 2017;11(11):Cd011564. doi:10.1002/14651858.CD011564.pub2

34. Hosny SS, Ali HM, Mohammed WA, El Ghannam MH. Study of relationship between total vitamin D level and NAFLD in a sample of Egyptian patients with and without T2DM. Diabetes Metab Syndr. 2019;13(3):1769-1771. doi:10.1016/j.dsx.2019.04.002

35. Wortsman J, Matsuoka LY, Chen TC, Lu Z, Holick MF. Decreased bioavailability of vitamin D in obesity. Am J Clin Nutr. 2000;72(3):690-693. doi:10.1093/ajcn/72.3.690

36. Flegal KM, Shepherd JA, Looker AC, et al. Comparisons of percentage body fat, body mass index, waist circumference, and waist-stature ratio in adults. Am J Clin Nutr. 2009;89(2):500-508. doi:10.3945/ajcn.2008.26847

37. Caleyachetty R, Barber TM, Mohammed NI, et al. Ethnicity-specific BMI cutoffs for obesity based on type 2 diabetes risk in England: a population-based cohort study. Lancet Diabetes Endocrinol. 2021;9(7):419-426. doi:10.1016/S2213-8587(21)00088-7

38. Teufel F, Seiglie JA, Geldsetzer P, et al. Body-mass index and diabetes risk in 57 low-income and middle-income countries: a cross-sectional study of nationally representative, individual-level data in 685616 adults. Lancet. 2021;398(10296):238-248. doi:10.1016/S0140-6736(21)00844-8

39. Li Y, Teng D, Shi X, et al. Prevalence of diabetes recorded in mainland China using 2018 diagnostic criteria from the American Diabetes Association: national cross sectional study. BMJ. 2020;369:m997. doi:10.1136/bmj.m997

40. Wang L, Zhou B, Zhao Z, et al. Body-mass index and obesity in urban and rural China: findings from consecutive nationally representative surveys during 2004-18. Lancet. 2021;398(10294):53-63. doi:10.1016/S0140-6736(21)00798-4

41. Pan XF, Wang L, Pan A. Epidemiology and determinants of obesity in China. Lancet Diabetes Endocrinol. 2021;9(6):373-392. doi:10.1016/S2213$8587(21) 00045-0$

42. Wang Q, Shi X, Wang J, Zhang J, Xu C. Low serum vitamin D concentrations are associated with obese but not lean NAFLD: a cross-sectional study. Nutr J. 2021;20(1):30. doi:10.1186/s12937-021-00690-9

43. Barchetta I, Angelico F, Del Ben M, et al. Strong association between non alcoholic fatty liver disease (NAFLD) and low 25(OH) vitamin D levels in an adult population with normal serum liver enzymes. BMC Med. 2011;9:85. doi:10.1186/1741-7015-9-85

44. Seo JA, Eun CR, Cho H, et al. Low vitamin D status is associated with nonalcoholic Fatty liver disease independent of visceral obesity in Korean adults. PLoS One. 2013;8(10):e75197. doi:10.1371/journal.pone.0075197

45. Nelson JE, Roth CL, Wilson LA, et al. Vitamin D deficiency is associated with increased risk of non-alcoholic steatohepatitis in adults with nonalcoholic fatty liver disease: possible role for MAPK and NF-кB? Am J Gastroenterol. 2016;111(6):852-863. doi:10.1038/ajg.2016.51

46. Farhangi MA, Mesgari-Abbasi M, Hajiluian G, Nameni G, Shahabi P. Adipose tissue inflammation and oxidative stress: the ameliorative effects of Vitamin D. Inflammation. 2017;40(5):1688-1697. doi:10.1007/s10753-017-0610-9

47. Asemi Z, Hashemi T, Karamali M, Samimi M, Esmaillzadeh A. Effects of vitamin D supplementation on glucose metabolism, lipid concentrations, inflammation, and oxidative stress in gestational diabetes: a double-blind randomized controlled clinical trial. Am J Clin Nutr. 2013;98 (6):1425-1432. doi:10.3945/ajen.113.072785

48. Mohammadi SM, Eghbali SA, Soheilikhah S, et al. The effects of vitamin D supplementation on adiponectin level and insulin resistance in first-degree relatives of subjects with type 2 diabetes: a randomized double-blinded controlled trial. Electron Phy. 2016;8(9):2849-2854. doi:10.19082/2849

49. Mutt SJ, Hyppönen E, Saarnio J, Järvelin MR, Herzig KH. Vitamin D and adipose tissue-more than storage. Front Physiol. 2014;5:228. doi: $10.3389 /$ fphys.2014.00228

50. Alhabeeb H, Kord-Varkaneh H, Tan SC, et al. The influence of omega-3 supplementation on vitamin D levels in humans: a systematic review and dose-response meta-analysis of randomized controlled trials. Crit Rev Food Sci Nutr. 2020;1-8. doi:10.1080/10408398.2020.1863905

51. Talaei A, Mohamadi M, Adgi Z. The effect of vitamin D on insulin resistance in patients with type 2 diabetes. Diabetol Metab Syndr. 2013;5(1):8. doi:10.1186/1758-5996-5-8

52. Papapostoli I, Lammert F, Stokes CS. Effect of short-term Vitamin D correction on hepatic steatosis as quantified by controlled attenuation parameter (CAP). J Gastrointestin Liver Dis. 2016;25(2):175-181. doi:10.15403/jgld.2014.1121.252.cap

53. Heaney RP. Vitamin D-baseline status and effective dose. $N$ Engl J Med. 2012;367(1):77-78. doi:10.1056/NEJMe1206858

54. Rezaei S, Tabrizi R, Nowrouzi-Sohrabi P, et al. The effects of Vitamin D supplementation on anthropometric and biochemical indices in patients with non-alcoholic fatty liver disease: a systematic review and meta-analysis. Front Pharmacol. 2021;12:732496. doi:10.3389/fphar.2021.732496

55. Gheorghe G, Bungău S, Ceobanu G, et al. The non-invasive assessment of hepatic fibrosis. J Formos Med Assoc. 2021;120(2):794-803. doi:10.1016/j.jfma.2020.08.019

56. Zhang Y, Jiang X, Li X, et al. Serum Vitamin D levels and risk of liver cancer: a systematic review and dose-response meta-analysis of cohort studies. Nutr Cancer. 2021;73(8):1-9. doi:10.1080/01635581.2020.1797127

57. Benedict M, Zhang X. Non-alcoholic fatty liver disease: an expanded review. World J Hepatol. 2017;9(16):715-732. doi:10.4254/wjh.v9.i16.715 58. Day CP, James OF. Steatohepatitis: a tale of two "hits"? Gastroenterology. 1998;114(4):842-845. doi:10.1016/S0016-5085(98)70599-2 
59. Zhou QG, Hou FF, Guo ZJ, Liang M, Wang GB, Zhang X. 1,25-Dihydroxyvitamin D improved the free fatty-acid-induced insulin resistance in cultured C2C12 cells. Diabetes Metab Res Rev. 2008;24(6):459-464. doi:10.1002/dmrr.873

60. Maia-Ceciliano TC, Dutra RR, Aguila MB, Mandarim-de-lacerda CA. The deficiency and the supplementation of vitamin D and liver: lessons of chronic fructose-rich diet in mice. J Steroid Biochem Mol Biol. 2019;192:105399. doi:10.1016/j.jsbmb.2019.105399

61. Tamadon MR, Soleimani A, Keneshlou F, et al. Clinical trial on the effects of Vitamin D supplementation on metabolic profiles in diabetic hemodialysis. Horm Metab Res. 2018;50(1):50-55. doi:10.1055/s-0043-119221

62. Tarcin O, Yavuz DG, Ozben B, et al. Effect of vitamin D deficiency and replacement on endothelial function in asymptomatic subjects. $J$ Clin Endocrinol Metab. 2009;94(10):4023-4030. doi:10.1210/jc.2008-1212

63. Eftekhari MH, Akbarzadeh M, Dabbaghmanesh MH, Hassanzadeh J. The effect of calcitriol on lipid profile and oxidative stress in hyperlipidemic patients with type 2 diabetes mellitus. ARYA Atheroscler. 2014;10(2):82-88.

64. Nakano T, Cheng YF, Lai CY, et al. Impact of artificial sunlight therapy on the progress of non-alcoholic fatty liver disease in rats. $J$ Hepatol. 2011;55(2):415-425. doi:10.1016/j.jhep.2010.11.028

65. Caldwell SH, Argo CK. Divergent effects of peroxisome proliferator-activated receptor-gamma ligands in human and mouse nonalcoholic steatohepatitis. Hepatology. 2007;46(2):285-287. doi:10.1002/hep.21881

66. Haussler MR, Haussler CA, Bartik L, et al. Vitamin D receptor: molecular signaling and actions of nutritional ligands in disease prevention. Nutr Rev. 2008;66(10 Suppl 2):S98-112. doi:10.1111/j.1753-4887.2008.00093.x

67. Ford ES, Ajani UA, McGuire LC, Liu S. Concentrations of serum vitamin D and the metabolic syndrome among U.S. adults. Diabetes Care. 2005;28(5):1228-1230. doi:10.2337/diacare.28.5.1228

68. Abenavoli L, Milic N, Di Renzo L, Preveden T, Medić-Stojanoska M, De Lorenzo A. Metabolic aspects of adult patients with nonalcoholic fatty liver disease. World j Gastroenterol. 2016;22(31):7006-7016. doi:10.3748/wjg.v22.i31.7006

69. Kong M, Zhu L, Bai L, et al. Vitamin D deficiency promotes nonalcoholic steatohepatitis through impaired enterohepatic circulation in animal model. Am J Physiol Gastrointest Liver Physiol. 2014;307(9):G883-893. doi:10.1152/ajpgi.00427.2013

70. Ebrahimzadeh Leylabadlo H, Samadi Kafil H, Farajnia S, et al. Gut microbiota in nonalcoholic fatty liver diseases with and without type-2 diabetes mellitus. Eur J Gastroenterol Hepatol. 2021;33:e548-e554. doi:10.1097/MEG.0000000000002140

Diabetes, Metabolic Syndrome and Obesity: Targets and Therapy

\section{Dovepress}

\section{Publish your work in this journal}

Diabetes, Metabolic Syndrome and Obesity: Targets and Therapy is an international, peer-reviewed open-access journal committed to the rapid publication of the latest laboratory and clinical findings in the fields of diabetes, metabolic syndrome and obesity research. Original research, review, case reports, hypothesis formation, expert opinion and commentaries are all considered for publication. The manuscript management system is completely online and includes a very quick and fair peer-review system, which is all easy to use. Visit http://www.dovepress. com/testimonials.php to read real quotes from published authors.

Submit your manuscript here: https://www.dovepress.com/diabetes-metabolic-syndrome-and-obesity-targets-and-therapy-journal 